\title{
Spectroscopic study of the elusive globular cluster ESO452-SC11 and its surroundings ${ }^{\star}$
}

\author{
Andreas Koch ${ }^{1}$, Camilla Juul Hansen ${ }^{2}$, and Andrea Kunder ${ }^{3,4}$ \\ 1 Department of Physics, Lancaster University, LA1 4YB, Lancaster, UK \\ e-mail: a.koch1@lancaster.ac.uk \\ 2 Dark Cosmology Centre, Niels Bohr Institute, University of Copenhagen, Juliane Maries Vej 30, 2100 Copenhagen, Denmark \\ 3 Leibniz-Institut für Astrophysik Potsdam, An der Sternwarte 16, 14482 Potsdam, Germany \\ 4 Saint Martin's University, Old Main, 5000 Abbey Way SE, Lacey, WA 98503, USA
}

Received 14 March 2017 / Accepted 24 April 2017

\begin{abstract}
Globular clusters (GCs) have long been recognized as being amongst the oldest objects in the Galaxy. As such, they have the potential of playing a pivotal role in deciphering the Milky Way's early history. Here we present the first spectroscopic study of the lowmass system ESO452-SC11 using the AAOmega multifibre spectrograph at medium resolution. Given the stellar sparsity of this object and the high degree of foreground contamination due to its location toward the Galactic bulge, very few details are known for this cluster - there is no consensus, for instance, about its age, metallicity, or its association with the disk or bulge. We identify five member candidates based on common radial velocity, calcium-triplet metallicity, and position within the GC. Using spectral synthesis, the measurement of accurate $\mathrm{Fe}$-abundances from Fe-lines, and abundances of several $\alpha$-, Fe-peak, and neutron-capture elements (Si, $\mathrm{Ca}, \mathrm{Ti}, \mathrm{Cr}, \mathrm{Co}, \mathrm{Ni}, \mathrm{Sr}$, and $\mathrm{Eu}$ ) is carried out, albeit with large uncertainties. We find that two of the five cluster candidates are likely non-members, as they have deviating iron abundances and $[\alpha / \mathrm{Fe}]$ ratios. The cluster mean heliocentric velocity is $19 \pm 2 \mathrm{~km} \mathrm{~s}^{-1}$ with a velocity dispersion of $2.8 \pm 3.4 \mathrm{~km} \mathrm{~s}^{-1}$, a low value in line with its sparse nature and low mass. The mean Fe-abundance from spectral fitting is $-0.88 \pm 0.03 \mathrm{dex}$, where the spread is driven by observational errors. Furthermore, the $\alpha$-elements of the GC candidates are marginally lower than expected for the bulge at similar metallicities. As spectra of hundreds of stars were collected in a 2-degree field centered on ESO452-SC11, a detailed abundance study of the surrounding field was also enabled. The majority of the non-members have slightly higher $[\alpha / \mathrm{Fe}]$ ratios, in line with the typical nearby bulge population. A subset of the spectra with measured Fe-peak abundance ratios shows a large scatter around solar values, albeit with large uncertainties. Furthermore, our study provides the first systematic measurements of strontium abundances in a Galactic bulge GC. Here, the Eu and Sr abundances of the GC candidates are broadly consistent with a disk or bulge association. Recent proper motions and our orbital calculations place ESO452 on an elliptical orbit in the central $3 \mathrm{kpc}$ of the Milky Way, establishing a firm connection with the bulge. Finally, while the radial velocities and preferential position of a dozen of stars outside the GC radius appear to imply the presence of extra-tidal stars, their significantly different chemical composition refutes this hypothesis.
\end{abstract}

Key words. stars: abundances - Galaxy: abundances - Galaxy: structure - globular clusters: individual: ESO452-SC11 Galaxy: disk - Galaxy: bulge

\section{Introduction}

Star clusters, in particular the old globular clusters (GCs), are important probes of the formation and structure of our Galaxy. Especially when studying objects toward the Milky Way central regions, the interface of various Galactic components becomes important, where the bulge, thin and thick disks, and the halo tend to overlap (e.g., Chiba \& Beers 2000; Koch \& McWilliam 2008; Ness et al. 2013). While it is difficult to distinguish these structures purely based on their metallicity or kinematics, chemical abundance tagging provides a powerful tool to study the evolution and distinction of the individual components in detail (e.g., Nissen \& Schuster 2010; McWilliam 2016).

ESO452-SC11 (in the following ESO452 for brevity) is a poorly studied object located at $\sim 2 \mathrm{kpc}$ from the Galactic Center at low latitude that was discovered in the ESO/Uppsala B survey of Lauberts et al. (1981). Table 1 lists the fundamental properties

* Full Tables 2-5 are available at the CDS via anonymous ftp to cdsarc.u-strasbg. fr (130.79.128.5) or via

http://cdsarc.u-strasbg.fr/viz-bin/qcat?]/A+A/604/A41 of this GC. The recent study of Cornish et al. (2006) found that depending on the exact isochrones used, the age of this GC was still difficult to constrain, leaving a possible range between 9 and 16 Gyr. Similarly, a metallicity between -1.4 and -0.4 dex and heliocentric distances between 6.6 and $7.5 \mathrm{kpc}$ were in the permitted parameter space of this sparse object's color-magnitude diagram (CMD). Any conclusions on its properties were also sensitive to the $\alpha$-enhancement of the isochrones in the fitting of Cornish et al. (2006).

Within this broad parameter range, ESO452 was placed within the bulge-halo transition, at the lower end of the bulge metallicity distribution function (MDF), at a similar age to "somewhat young halo clusters" (Cornish et al. 2006). It has therefore been suggested that this unique GC could have been placed within the bulge by an interaction with a satellite galaxy (see also Marín-Franch et al. 2009; Ferraro et al. 2009), which still needs to be consolidated by a chemodynamical study of ESO452.

Here, we report on our large-field-of-view $\left(2^{\circ}\right)$, multi-object spectroscopic effort with the AAOmega spectrograph to capture 
Table 1. Properties of ESO452-SC11 from the literature and this work.

\begin{tabular}{ccc}
\hline \hline Parameter & Value & Reference \\
\hline$(\alpha, \delta)(\mathrm{J} 2000.0)$ & $(16: 39: 25.45,-28: 23: 55.3)$ & $(1)$ \\
$(l, b)$ & $\left(351.91^{\circ},+12.10^{\circ}\right)$ & $(1)$ \\
$R_{\mathrm{GC}}, R_{\odot}$ & $\sim 2 \mathrm{kpc}, \sim 7 \mathrm{kpc}$ & $(1),(2)$ \\
$r_{\mathrm{c}}, r_{\mathrm{h}}, r_{\mathrm{t}}$ & $0.5^{\prime}, 0.5^{\prime}, 5.0^{\prime}$ & $(1)$ \\
$M_{V}$ & $-4.02 \mathrm{mag}$ & $(1)$ \\
$E(B-V)$ & $0.521 \mathrm{mag}, 0.448 \mathrm{mag}^{\prime}$ & $(3),(4)$ \\
$\left\langle v_{\mathrm{HC}}\right\rangle, \sigma$ & $19 \pm 2 \mathrm{~km} \mathrm{~s}^{-1}, 2.8 \pm 3.4 \mathrm{~km} \mathrm{~s}^{-1}$ & $(5)$ \\
$\langle[\mathrm{Fe} / \mathrm{H}]\rangle_{\left(\mathrm{SP} \_\mathrm{ACE}\right)}$ & $-0.88 \mathrm{dex}$ & $(5)$ \\
\hline
\end{tabular}

References. (1) Harris (1996, 2010 version); (2) Cornish et al. (2006); (3) Schlegel et al. (1998); (4) Schlafly \& Finkbeiner (2011); (5) This work.

this elusive object. Our main focus thus lies on establishing membership with the GC and on kinematically and chemically characterizing it, also in the context of the surrounding field. This paper is organized as follows: in Sect. 2 we introduce our spectroscopic data set, and in Sect. 3 we use kinematic and metallicity information to assess stars that are probable members of the GC. Section 4 is dedicated to a stellar parameter and abundance analysis of the cluster stars and the foreground component, while in Sects. 5 and 6 we investigate possible correlations of our abundance results with kinematics and test for the presence of extra-tidal stars. In Sect. 7 we conclude.

\section{Observations and data reduction}

Prompted by the large field of view of our pointings, we opted to select targets from the $\mathrm{GC}\left(J-K_{\mathrm{S}}, K_{\mathrm{S}}\right) \mathrm{CMD}$ (see Fig. 1) that we assembled from the Two Micron All Sky Survey (2MASS; Cutri et al. 2003). Selection boxes were drawn encompassing the cluster red giant branch (RGB) from the tip RGB to $\sim 3$ mag fainter, thus approximately one magnitude above its red clump. Furthermore, priority was given to stars with small radial distances to the nominal GC center so as to maximize the number of member stars. This is only marginally efficient because of the overall small tidal radius of $r_{\mathrm{t}}=5^{\prime}$ of ESO452 (Table 1) and the sparse nature of this object. Furthermore, at the low Galactic latitude of $+12^{\circ}$, the yield of this procedure is still hampered by a large contamination with Galactic foreground stars.

All our data were taken on June 08, 2013, with the AAOmega multifibre spectrograph at the $3.9 \mathrm{~m}$ Anglo-Australian Telescope (Siding Spring, Australia). A single mask was created for the Two Degree Field (2dF) fibre positioner, containing 363 science fibres and 25 sky positions. The dual setup we used employed the blue $3200 \mathrm{~B}$ grating, centered at $4100 \AA$, and the red $1700 \mathrm{D}$ grating, centered at $8600 \AA$, so as to comprise the neutron-capture elements $\mathrm{Sr}$ and $\mathrm{Eu}$ in the blue (Hansen et al. 2015) and the prominent calcium triplet $(\mathrm{CaT})$ lines in the red. The exposure time was chosen as $4 \times 20 \mathrm{~min}$. The data were reduced using the 2dfdr pipeline provided by the AAO, which performs standard reduction steps from quartz-flatfielding and wavelength calibration via arc-lamp exposures, sky subtraction using the dedicated sky fibres, to optimal extraction of the science spectra. The final spectra cover the wavelength ranges of 3970-4250 and 8350$8800 \AA$, respectively, with slight variations depending on the exact location of the spectra on the CCD. The resolving power of the blue and red spectra is $\sim 9000$ and 10600 , as measured from the width of the calibration arc lamps. Finally, the median signalto-noise ratio $(\mathrm{S} / \mathrm{N})$ of our final extracted spectra is $15 \mathrm{px}^{-1}$ in the

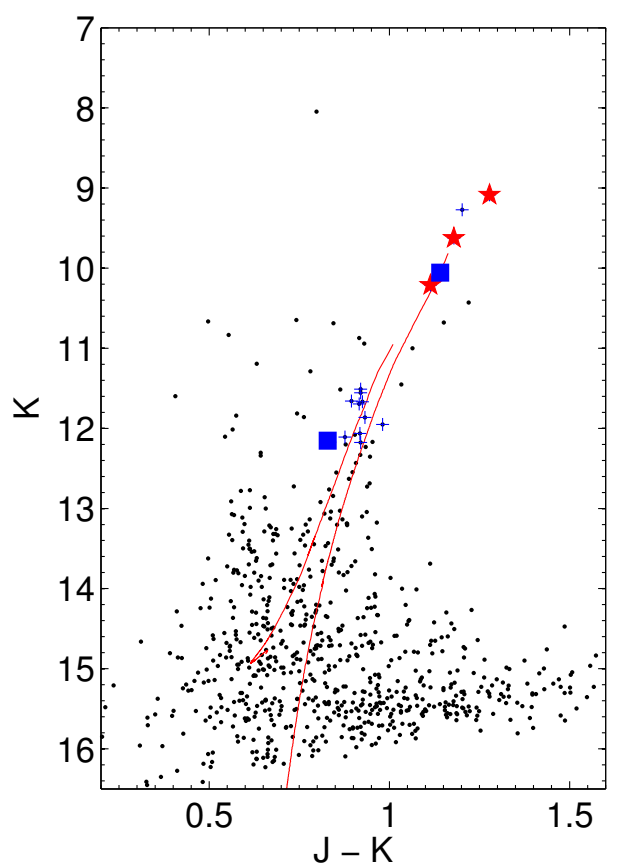

Fig. 1. 2MASS CMD of ESO452-SC11 within its tidal radius. Targets within this radius are shown as blue crosses, red symbols denote our most central bona fide member candidates, and the blue squares are two additional candidates with similar kinematics but different chemistry. Also shown is a Dartmouth isochrone (Dotter et al. 2008) with the cluster parameters from the literature and as found in this work.

blue and $50 \mathrm{px}^{-1}$ on the red chip, respectively. While the blue spectra reach $\mathrm{S} / \mathrm{Ns}$ up to $20 \mathrm{px}^{-1}$, the values on the red CCDs reach as high as $170 \mathrm{px}^{-1}$.

\section{Radial velocities, metallicities, and membership}

In the following, we assess the membership, metallicity, and basic kinematic properties that can be derived from our spectra.

\subsection{Radial velocity}

Individual heliocentric radial velocities of the target stars were determined via cross-correlation of the three prominent CaT lines against a Gaussian template, using the IRAF fxcor task (e.g., Kleyna et al. 2004). The typical median velocity error achieved in this way is $1.4 \mathrm{~km} \mathrm{~s}^{-1}$. Measurements of individual stars are listed in Table 2, and Fig. 2 shows the run of the heliocentric radial velocities with radial distance from the center of ESO452.

Guided by the very similar velocities of the two innermost stars, well within the half-light radius, we identify five stars within the tidal radius at similar velocity with individual values that lie between 14.2 and $20.5 \mathrm{~km} \mathrm{~s}^{-1}$. All other stars within this GC radius deviate by more than $15 \mathrm{~km} \mathrm{~s}^{-1}$ from these candidates, so that we define these five objects as the cluster population based on their kinematics and position. The mean systemic velocity of the five candidate members is $17.5 \pm 1.1 \mathrm{~km} \mathrm{~s}^{-1}$ and their radial velocity dispersion is low, at $2.1 \pm 1.0 \mathrm{~km} \mathrm{~s}^{-1}$, which is fully in line with the low mass of the cluster (Pryor \& Meylan 1993; Dubath et al. 1997).

In Sect. 4 we show that it is advisable to cull two candidates based on their $[\mathrm{Fe} / \mathrm{H}]$ and $[\alpha / \mathrm{Fe}]$ ratios because these are significantly different from the remainder of the GC sample. 
Table 2. Properties of the target stars.

\begin{tabular}{rccccc}
\hline \hline $\operatorname{Star}^{a}$ & $\begin{array}{c}(\alpha, \delta) \\
(\mathrm{J} 2000.0)\end{array}$ & $\begin{array}{c}r^{b} \\
{\left[^{\prime}\right]}\end{array}$ & $\begin{array}{c}v_{\mathrm{HC}} \\
{\left[\mathrm{km} \mathrm{s}^{-1}\right]}\end{array}$ & $\begin{array}{c}\Sigma E W(\mathrm{CaT}) \\
{[\mathrm{mA}]}\end{array}$ & $\begin{array}{c}{[\mathrm{Fe} / \mathrm{H}]_{\mathrm{CaT}}} \\
{[\mathrm{dex}]}\end{array}$ \\
\hline \multicolumn{7}{c}{ Member candidates } \\
\hline 55 & $16: 39: 27.9-28: 24: 07.1$ & 0.34 & $20.0 \pm 1.4$ & $6.19 \pm 0.09$ & $-1.15 \pm 0.20$ \\
56 & $16: 39: 38.9-28: 26: 20.8$ & 2.84 & $14.6 \pm 1.2$ & $5.74 \pm 0.21$ & $-1.01 \pm 0.22$ \\
204 & $16: 39: 24.1-28: 23: 07.6$ & 0.81 & $17.7 \pm 1.3$ & $6.29 \pm 0.11$ & $-1.22 \pm 0.20$ \\
362 & $16: 39: 25.3-28: 23: 38.5$ & 0.28 & $20.5 \pm 1.5$ & $6.45 \pm 0.91$ & $-1.24 \pm 0.44$ \\
396 & $16: 39: 44.3-28: 25: 10.9$ & 2.42 & $14.2 \pm 1.8$ & $6.34 \pm 0.13$ & $-1.11 \pm 0.21$ \\
\hline \multicolumn{7}{c}{ Fore- and background candidates } \\
\hline 2 & $16: 41: 47.8-28: 25: 02.2$ & 15.7 & $-12.2 \pm 1.6$ & $5.56 \pm 0.14$ & $\ldots$ \\
\hline
\end{tabular}

Notes. Table 2 is available in its entirety in electronic form at the CDS. ${ }^{(a)}$ IDs corresponding to our fibre numbers. ${ }^{(b)}$ Radial distance from the nominal cluster center.

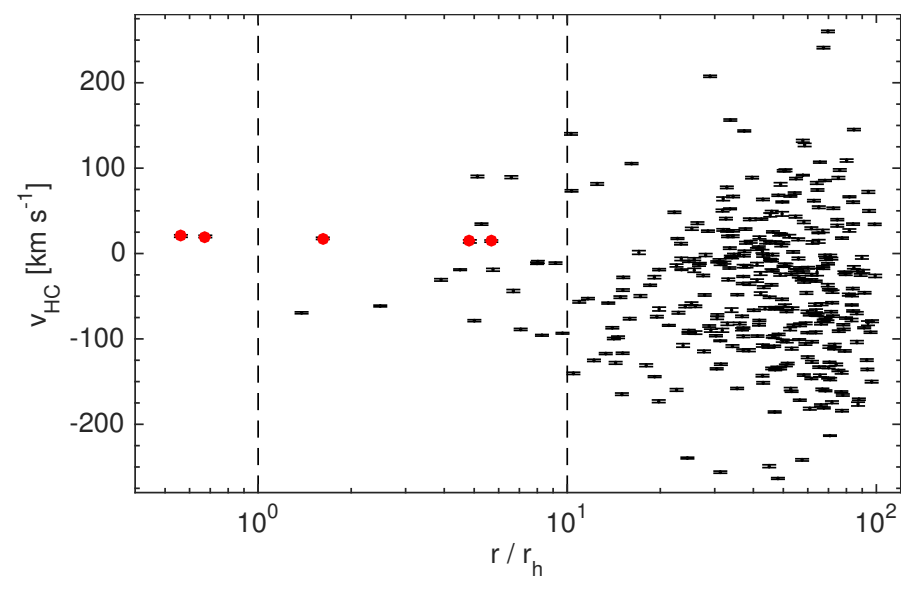

Fig. 2. Heliocentric radial velocities versus radial distance from the cluster center in units of its half-light radius. The dashed lines illustrate the GC half-light and nominal tidal radius. Our five bona fide candidates are highlighted with red circles.

Retaining only the three best candidates, a mean velocity of $19.4 \pm 1.8 \mathrm{~km} \mathrm{~s}^{-1}$ is found with a dispersion of $2.8 \pm 3.4 \mathrm{~km} \mathrm{~s}^{-1}$. As these three stars are those that are located closest to the cluster center, while the two most deviant stars lie at $2.5^{\prime}$ - five halflight radii away from the GC center - it is fair to adopt these three stars as the most probable cluster members.

\subsection{Calcium triplet metallicity}

The prominent near-infrared CaT lines at $\sim 8500 \AA$ are powerful indicators of the global metallicity in red giants (e.g., Armandroff \& Zinn 1988). Generally starting from some linear combinations of the line equivalent widths (EWs), the stars' luminosity also needs to be accounted for in order to remove higher order dependencies on surface gravity. While this is usually done in terms of the $V$-band magnitude above the horizontal branch $\left(V-V_{\mathrm{HB}}\right)$, our work employed infrared colors throughout, making this standard optical approach poorly calibrated. Infrared (2MASS) $K_{\mathrm{S}}$-magnitudes instead of $V$ - or $I$-data have more advantages, since $K_{\mathrm{S}}$ is less sensitive to reddening; thus, any dependence of the reduced width, $W^{\prime}$, on intracluster reddening is reduced (Warren \& Cole 2009).
Various calibrations of the metallicity scale based on infrared magnitudes have been proposed (e.g., Warren \& Cole 2009; Mauro et al. 2014; Vásquez et al. 2015). Here, we chose to adopt the study of Vásquez et al. (2015), which is based on Galactic bulge stars, as it covers a broad range in metallicity from -2.3 to 0.7 dex. These calibrations to the metallicity scale of Carretta et al. (2009) read

$[\mathrm{Fe} / \mathrm{H}]=-3.15+0.432 W^{\prime}+0.006 W^{\prime 2}$,

where the reduced width $W^{\prime}=\Sigma E W+0.384\left(K-K_{\mathrm{RC}}\right)$ and the CaT line strength was defined as the straight sum of the two strongest lines, $\Sigma E W=E W_{8542}+E W_{8662}$. Errors on EW measurements and photometry were propagated through this formalism and added in quadrature to the RMS scatter of 0.19 dex in the calibrations, as evaluated by Vásquez et al. (2015).

One important issue pertains to the magnitude of the red clump, $K_{\mathrm{RC}}$. Our $2 \mathrm{MASS}$ CMD and a suitable set of old moderately metal-poor isochrones ( -1 dex Dotter et al. 2008) show a sparse red clump at $K_{\mathrm{S}}=15 \mathrm{mag}$, which we adopt as the best value in our subsequent metallicity calibration. However, the optical data of Cornish et al. (2006) imply distance moduli that vary by 0.6 mag depending on the metallicity and $\alpha$ enhancement used in their isochrone fitting. Here, we account for such uncertainties by also testing the CaT calibration with a fainter red clump level of $K_{\mathrm{S}}=15.6 \mathrm{mag}$. This would systematically lower the resulting metallicity scale by $0.11 \mathrm{dex}$.

The resulting mean metallicity from the CaT of the five member candidates is -1.15 dex with a $1 \sigma$ scatter of 0.09 dex. As above, we consider the three most central stars as best members, and obtain a mean CaT metallicity of -1.19 dex (scatter of $0.05 \mathrm{dex}$ ) for them. Comparison with the median measurement error of 0.20 dex indicates that this scatter in the metallicities is most likely only due to the uncertainty in our CaT determination and is not an intrinsic spread, in line with the very low overall mass of the system (e.g., Koch et al. 2012). We did not attempt to assign any metallicities from the CaT to the Galactic contaminants, since the above calibrations are only valid at the assumed red clump magnitude, thus the distance of the GC. The Galactic stars, in turn, are located at random unknown distances to the observer.

The CMD study of Cornish et al. (2006) attempted isochrone fits of ESO452, but the sparse nature of this object left room 
Table 3. Stellar parameters from our photometric analysis and from SP_ACE for the member candidates in ESO4522-SC11.

\begin{tabular}{|c|c|c|c|c|c|c|c|}
\hline \multirow[t]{2}{*}{ Star } & \multirow[t]{2}{*}{$J-K$} & \multicolumn{2}{|c|}{$T_{\text {eff }}[K]$} & \multirow[t]{2}{*}{$\log g$} & \multirow{2}{*}{$\begin{array}{c}\xi \\
{\left[\mathrm{km} \mathrm{s}^{-1}\right]}\end{array}$} & \multirow[t]{2}{*}{$S / N$} & \multirow[t]{2}{*}{$\chi^{2}$} \\
\hline & & Alonso & SP_ACE & & & & \\
\hline 55 & 1.114 & 3982 & $3983_{-}^{+}$ & 1.64 & 1.47 & 86 & 1.13 \\
\hline 56 & 0.829 & 4619 & 479 & $3.30_{-0.28}^{+0.47}$ & 1.24 & 37 & 1.05 \\
\hline 204 & 1.179 & 3870 & $3928_{-43}^{+90}$ & $1.04_{-0.13}^{+0.51}$ & 1.47 & 110 & 1.10 \\
\hline 362 & 1.278 & $\cdots$ & $3880_{-31}^{+80}$ & $1.11_{-0.17}^{+0.36}$ & 1.42 & 91 & 1.03 \\
\hline 396 & 1.141 & 3910 & $3978_{-63}^{+61}$ & $1.63_{-0.43}^{+0.17}$ & 1.47 & 84 & 1.00 \\
\hline
\end{tabular}

Notes. Table 3 is available in its entirety at the CDS, including the fore- and background stars.

Table 4. Stellar abundances from SP_ACE for the member candidates in ESO4522-SC11.

\begin{tabular}{rrccccccccccc}
\hline \hline Star & {$[\mathrm{Fe} / \mathrm{H}]$} & $\sigma_{\mathrm{Fe}}$ & $N_{\mathrm{Fe}}$ & {$[\mathrm{Si} / \mathrm{Fe}]$} & $\sigma_{\mathrm{Si}}$ & $N_{\mathrm{Si}}$ & {$[\mathrm{Ca} / \mathrm{Fe}]$} & $\sigma_{\mathrm{Ca}}$ & $N_{\mathrm{Ca}}$ & {$[\mathrm{Ti} / \mathrm{Fe}]$} & $\sigma_{\mathrm{Ti}}$ & $N_{\mathrm{Ti}}$ \\
\hline 55 & -0.96 & \pm 0.05 & 3 & 0.75 & \pm 0.10 & 3 & 0.37 & ${ }_{-0.20}^{+0.35}$ & 4 & 0.03 & ${ }_{-0.14}^{+0.27}$ & 1 \\
56 & 0.15 & \pm 0.08 & 4 & -0.03 & \pm 0.14 & 1 & 0.18 & ${ }_{-0.23}^{+0.28}$ & 4 & -0.04 & \pm 0.24 & 1 \\
204 & -0.85 & \pm 0.05 & 4 & 0.44 & \pm 0.15 & 7 & 0.11 & ${ }_{-0.27}^{+0.38}$ & 4 & 0.05 & \pm 0.10 & 1 \\
362 & -0.82 & \pm 0.05 & 4 & 0.39 & \pm 0.16 & 5 & 0.14 & ${ }_{-0.23}^{+0.38}$ & 4 & 0.11 & ${ }_{-0.23}^{+0.06}$ & 1 \\
396 & -0.52 & \pm 0.05 & 4 & 0.46 & ${ }_{-0.13}^{+0.21}$ & 6 & 0.12 & ${ }_{-0.18}^{+0.31}$ & 4 & 0.04 & ${ }_{-0.20}^{+0.09}$ & 1 \\
\hline
\end{tabular}

Notes. Table 4 is available in its entirety at the CDS, including the fore- and background stars.

for a wide range of possible matches, with the cluster metallicity reaching from -1.4 up to -0.4 dex. Our $\mathrm{CaT}$ metallicity of -1.15 dex places ESO452 well within this parameter space. This metallicity is typical of globular clusters in the bulge (see also Sect. 5) as well as the metal-weak thick disk. In the next sections we further refine our measurement based on spectral synthesis.

\section{Stellar parameters and chemical abundances}

The near-infrared region around the $\mathrm{CaT}$ at intermediate resolution contains a wealth of spectral information in the form of several temperature- and gravity-sensitive features and chemical abundance tracers (Zwitter et al. 2004; Shetrone et al. 2009; Ruchti et al. 2010; Hendricks et al. 2014). Thus we also attempted to extract stellar parameters and chemical abundance ratios of several elements from the spectra in addition to the $\mathrm{CaT}$ metallicities we have obtained above.

\section{1. $S P \_A C E$}

To this end, we first used the SP_ACE code (Boeche \& Grebel 2016), which was originally designed to complement the Radial Velocity Experiment (RAVE) pipeline (Siebert et al. 2011; Kunder et al. 2017). RAVE operates in a similar wavelength region at slightly lower resolution than our data, but SP_ACE can been adapted to a much broader range of applicabilities.

In brief, EWs of each line in the input spectral region are first fit by a polynomial as a function of the stellar parameters $T_{\text {eff }}$, $\log g,[\mathrm{M} / \mathrm{H}]$, and $[\mathrm{X} / \mathrm{Fe}]$ for chemical elements $\mathrm{X}$. The actual SP_ACE routine then constructs curves of growth as a function of these parameters and determines the best fit in a $\chi^{2}$-sense. As detailed in Boeche \& Grebel (2016), the microturbulence is computed by SP_ACE ad hoc as a function of temperature and gravity (see also Koch et al. 2009; Jofré et al. 2014).

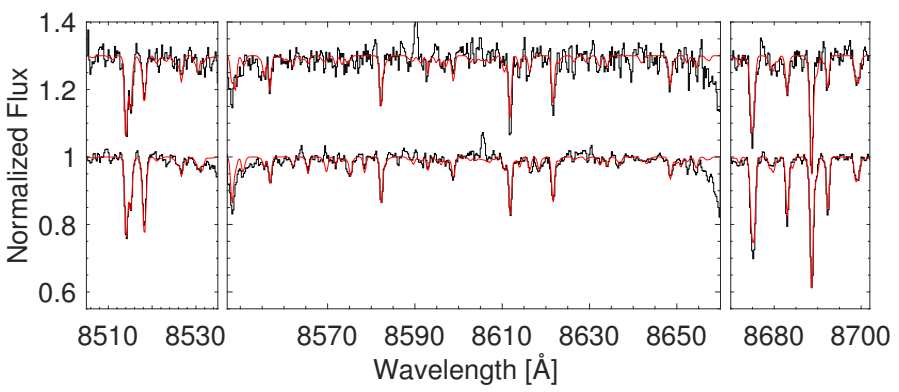

Fig. 3. Observed sample spectra (black) with the best-fit spectra from SP_ACE overlaid in red. Note the broken wavelength axis.

In practice, we restricted the spectral range to be fitted to 8505-8535, 8547-8660, and 8670-8702 A, thereby avoiding the strong $\mathrm{CaT}$ lines because of their very strong wings, which can cause difficulties in precise determinations of the surface gravity and/or the Ca-abundance of the stars. Furthermore, we thus avoided the TiO $\varepsilon$ band at $8432 \AA$ that can affect abundance analyses of cold metal-rich stars. To quantify the overall magnitude of its influence, we computed a $\mathrm{TiO} \varepsilon$ index following Sharples et al. (1990) and Kunder et al. (2012). As a result, this index is scattered around zero, which shows that the pseudocontinuum in this spectral range is predominantly flat and thus not affected by $\mathrm{TiO}$ absorption, as is expected because most our stars are warmer than $\sim 3800 \mathrm{~K}$ (see, e.g., Fig. 6 in Kunder et al. 2012) .

In the employed fitting region, up to eight Fe I plus II, nine Si I, 4 Ca I, nine Ti I, 3 Cr I, three Co I, and three Ni I features could be measured depending on the stellar type and the S/N of the spectra. Figure 3 shows two typical stars of our sample and the respective best-fit synthetic spectra.

All the stellar parameters of the member fore- or background stars thus derived are listed in Table 3, and the chemical 
Table 5. Same as Table 4, but for the elements Cr, Co, and Ni from SP_ACE.

\begin{tabular}{rccccccccccccc}
\hline \hline Star & {$[\mathrm{Cr} / \mathrm{Fe}]$} & $\sigma_{\mathrm{Cr}}$ & $N_{\mathrm{Cr}}$ & {$[\mathrm{Co} / \mathrm{Fe}]$} & $\sigma_{\mathrm{Co}}$ & $N_{\mathrm{Co}}$ & {$[\mathrm{Ni} / \mathrm{Fe}]$} & $\sigma_{\mathrm{Ni}}$ & $N_{\mathrm{Ni}}$ & {$[\mathrm{Sr} / \mathrm{Fe}]$} & $\sigma_{\mathrm{Sr}}$ & {$[\mathrm{Eu} / \mathrm{Fe}]$} & $\sigma_{\mathrm{Eu}}$ \\
\hline 55 & 0.00 & ${ }_{-0.25}^{+0.45}$ & 3 & $\ldots$ & $\ldots$ & 0 & $\ldots$ & $\ldots$ & 0 & 0.20 & 0.20 & $\ldots$ & $\ldots$ \\
56 & -0.40 & \pm 0.50 & 3 & -0.20 & \pm 0.55 & 2 & -0.35 & \pm 0.80 & 2 & $\ldots$ & $\ldots$ & $\ldots$ & $\ldots$ \\
204 & -0.18 & \pm 0.23 & 3 & 0.31 & \pm 0.25 & 3 & -0.41 & \pm 0.56 & 2 & 0.00 & 0.09 & 0.40 & 0.14 \\
362 & -0.20 & \pm 0.23 & 3 & 0.45 & \pm 0.23 & 2 & -0.20 & \pm 0.51 & 2 & $\ldots$ & $\ldots$ & $\ldots$ & $\ldots$ \\
396 & -0.30 & \pm 0.26 & 3 & $\ldots$ & $\ldots$ & 0 & 0.16 & ${ }_{-0.33}^{+0.60}$ & 2 & 0.10 & 0.30 & $>-0.30$ & $\ldots$ \\
\hline
\end{tabular}

Notes. $\mathrm{Sr}$ and Eu abundances, in turn, are based on synthetic fits. Table 5 is available in its entirety at the CDS, including the fore- and background stars.

abundance ratios are given in Tables 4 and 5. Owing to the statistical method of abundance fitting in SP_ACE, the code returns asymmetric error bars, which we list in the tables as lower and upper bounds. Finally, the number of lines, N, detected in each spectrum is also tabulated.

\section{2. $T_{\text {eff }}$ and $\log g$ from photometry}

To test the robustness of the parameters obtained from SP_ACE, we attempted two additional independent methods to estimate the temperatures and gravities of the all the observed stars. The short spectral ranges from the two AAOmega settings make it hard to determine $[\mathrm{Fe} / \mathrm{H}]$ accurately, and therefore using the $J-K$ color-T calibration is advantageous owing to its low metallicity dependence.

The temperatures were solely based on 2MASS photometry, since we only have infrared $J, H$, and $K$ magnitudes at hand. To calculate the temperature, we used the color calibrations from Alonso et al. (1999), which are based on the infrared flux method (IRFM) for giants. Furthermore, $(J-K)$ is the color of the three IR colors with the lowest internal uncertainty (Alonso et al. 1996, 1999). Moreover, these authors also recommended the use of $(J-K)$ when the metallicities were uncertain. We adopted the mean $E(B-V)$ from Schlafly \& Finkbeiner (2011) and converted extinction coefficients $\mathrm{A}_{J}$ and $\mathrm{A}_{K}$ from the IRSA Dust database ${ }^{1}$ to deredden the $J-K$-colors. To calculate $T_{\text {eff }}$, we used the SP_ACE output for $[\mathrm{Fe} / \mathrm{H}]$. Owing to the null metallicity dependence in the IRFM $(J-K)$ color calibrations of Alonso et al. (1996, 1999), we obtain identical temperatures (within 1-2 K) if we were to assume a fixed value of -1 dex versus the metallicities computed with SP_ACE, unless the adopted metallicity would push the star outside the allowed metallicity bin in the calibration. Therefore, we did not further iterate the calculated temperatures because of uncertainties in $[\mathrm{Fe} / \mathrm{H}]$. We note that seven stars deviate strongly from the otherwise good correlation between the Alonso and SP_ACE temperatures. These stars fall outside the metallicity range covered by the IRFM $T_{\text {eff }}$-color calibration. Above $4800 \mathrm{~K}$, approximately 30 stars yield Alonso temperatures considerably lower than those derived by SP_ACE. These stars are warmer, but faint, and the vast majority of them have reddening values above $0.4 \mathrm{mag}$, and they are most likely poorly classified; we also note a slight increase in the temperature difference with increasing gravity.

Following this, we tested the empirical $\log g$ - $T_{\text {eff }}$-relation from Barklem et al. (2005) to estimate the gravities. However, their empirical formula was calibrated based on stars that are typically warmer than $4300 \mathrm{~K}$, while many of our targets are very cool objects $(<4300 \mathrm{~K})$, resulting in highly negative

\footnotetext{
1 http://irsa.ipac.caltech.edu/applications/DUST/
}

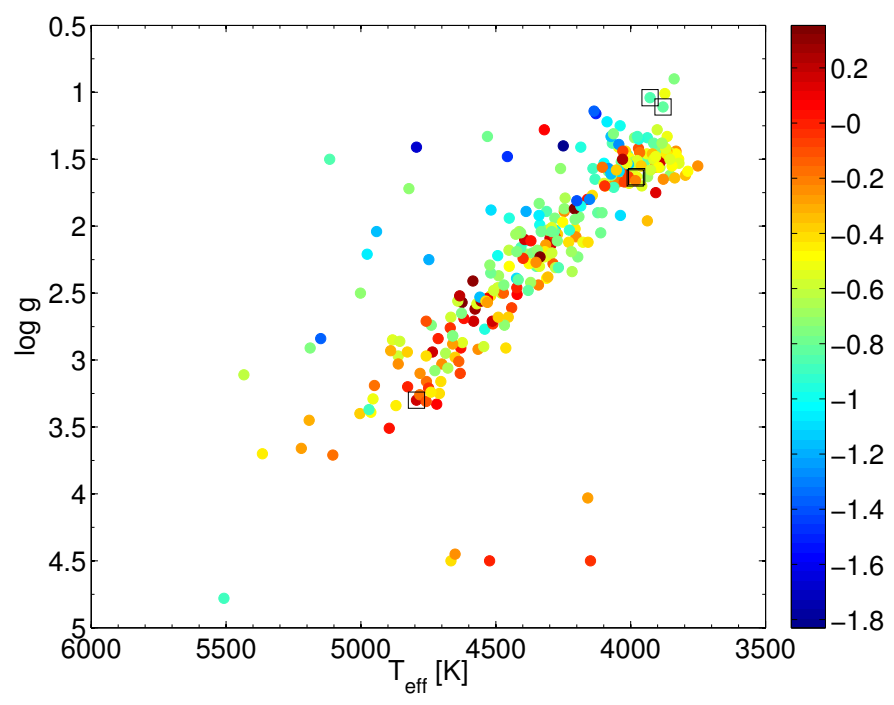

Fig. 4. Hertzsprung-Russell diagram of ESO452-SC11 with stellar parameters from SP_ACE, color-coded by iron abundance. The member candidates are highlighted with black squares. Note that two candidates with very similar stellar parameters overlap in this diagram.

gravities. Thus we discarded this approach and continued using the SP_ACE parameters described above.

\subsection{Final stellar parameters}

Figure 4 shows a Hertzsprung-Russel diagram of the entire target sample, color-coded by their iron abundance from SP_ACE. The RGB is well populated down to its base at $\log g \sim 3.5$ and the $T_{\text {eff-metallicity relation is indicated by a dozen metal-poor }}$ warmer giants. Furthermore, the foreground component contains a few metal-rich dwarf stars, typical of the Galactic disks. As a result of our color-selection criteria from the 2MASS, the majority of stars in the sample are therefore K-giants with temperatures lower than $\sim 5000 \mathrm{~K}$.

We note that one of our GC member candidates (number 56) has a considerably higher $\log g$, at $\sim 3.3 \mathrm{dex}$, compared to the values found for the other four, which lie at $\sim 1-1.6$ dex. While there is no reason why our selection criteria should not have picked stars at the base of the RGB, its deviating chemical abundance ratios indicate that this object may be a foreground star as well.

In Fig. 5 we compare the stellar temperatures obtained with the different methods outlined above. The calibrations of Alonso return $T_{\text {eff }}$ that are $62 \mathrm{~K}$ lower on average than the SP_ACE results, with a scatter of $109 \mathrm{~K}$ below $4500 \mathrm{~K}$. Above this temperature, the agreement worsens for the reasons discussed above. We 

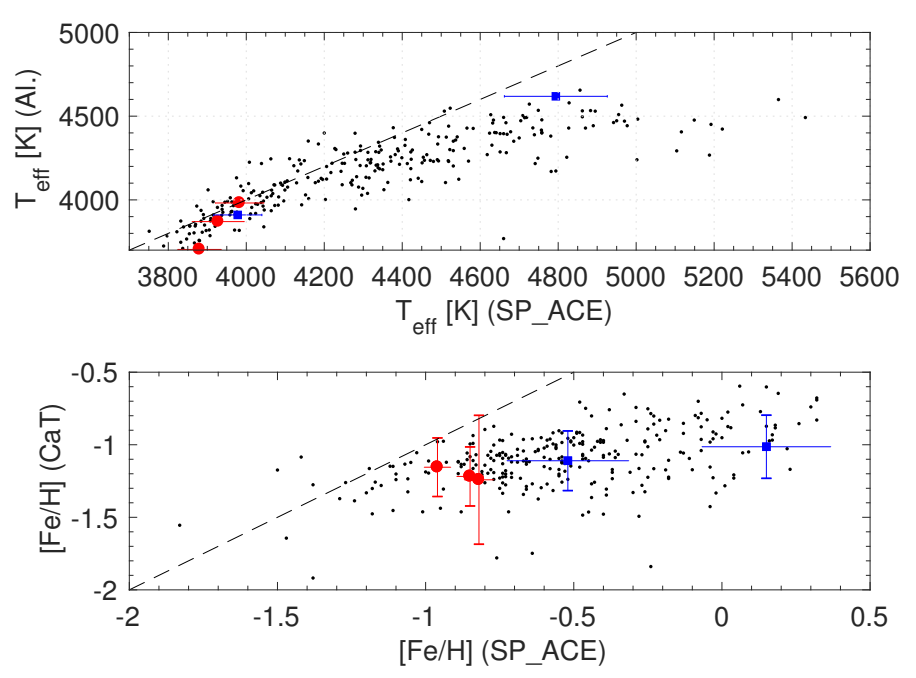

Fig. 5. Comparison of stellar parameters that were obtained through different methods. Red symbols show the innermost three member candidates, while blue symbols denote the two outer candidates with less secure membership. The dashed line shows unity.

note that the five GC candidates are in very good agreement with the Alonso scale. For the remainder of our analysis we adopt the stellar parameters from SP_ACE and note that although it is constrained to the short-wavelength coverage of the red AAOMega fibres, the code returns reliable values for $T_{\text {eff }}$ and more physically meaningful values for gravities and metallicities than any scaling formula we tested.

\subsection{Iron abundance}

In Fig. 5 (bottom panel) we compare the metallicities from the $\mathrm{CaT}$ with those obtained by the direct spectral analysis within SP_ACE. As noted above, CaT metallicities are meaningless for the foreground stars because the calibration cannot be applied for want of a reference red clump magnitude. The mean difference of the metallicity for the five GC member candidates from the Fe-lines and their CaT counterparts is 0.55 dex with a $1 \sigma$ scatter of 0.37 dex. Here, the CaT metallicities give systematically lower values and the difference is significant at the $1-3 \sigma$ level. In order to reconcile the measurements for the member stars, the $\mathrm{RC}$ magnitude would need to be lowered by $\sim 1.5$ mag; reddening uncertainties on that order of magnitude are unlikely. These differences, however, are driven by the two objects that are located farthest away from the GC center (stars 56 and 396), and as we show in Sect. 4.5. below, the higher-gravity object 56 has a high Fe-abundance of $0.15 \pm 0.08$ dex and also solar-scaled $[\alpha / \mathrm{Fe}]$ abundance ratios, suggesting that these two deviating objects are most likely foreground contaminants as well.

The mean Fe-abundance of the three bona fide GC member candidates (star IDs 55, 204, and 362) is $-0.88 \pm 0.03$ dex with a metallicity dispersion of $0.03 \pm 0.04 \mathrm{dex}$; this is higher than the value predicted by the CaT by 0.31 dex. As for the radial velocity dispersion of this system, also the very low spread in $\mathrm{Fe}$-abundances is fully compatible with the low mass of ESO452 (Carretta et al. 2009; Koch et al. 2012).

Figure 6 in turn shows the MDF from our entire sample, ranging from the metal-poor regime $([\mathrm{Fe} / \mathrm{H}] \sim-1.8)$ to slightly supersolar values of $\sim 0.3 \mathrm{dex}$. In that regard, the field surrounding ESO452 shows the typical shape and range for its location toward the Galactic bulge, including contributions from all nearby major subcomponents, including an inner halo, metal-weak thick

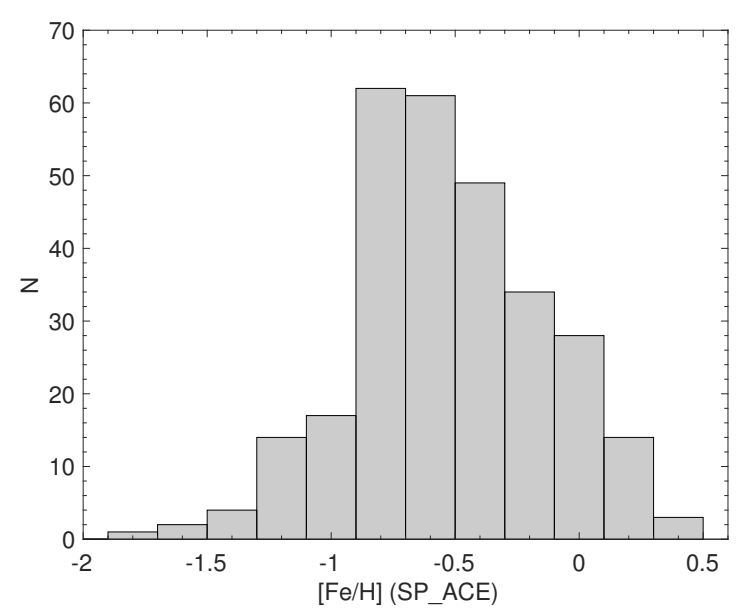

Fig. 6. Metallicity distribution of the entire foreground plus GC sample, using the Fe-abundances derived from SP_ACE.

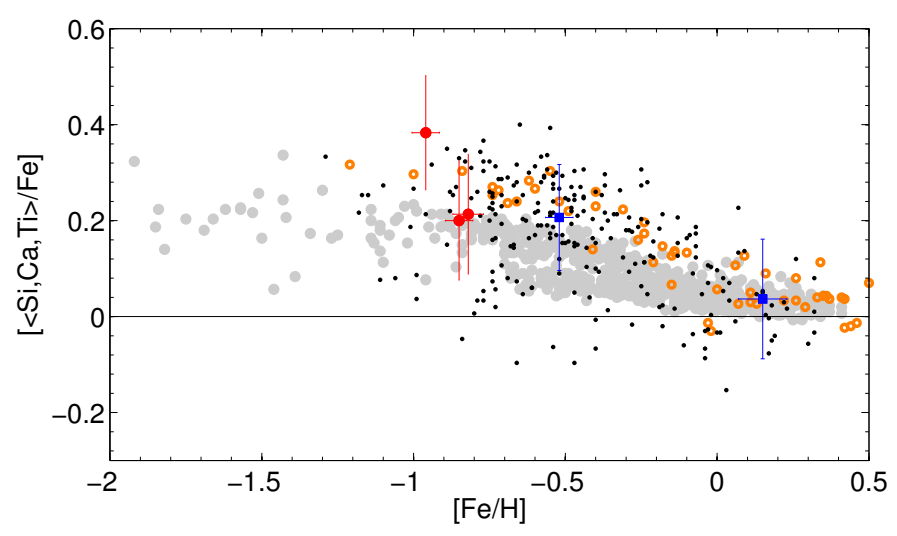

Fig. 7. Averaged $\alpha$-element abundances for our sample (black dots) in comparison with stars in the solar neighborhood from Bensby et al. (2014, light gray shade) and in the Galactic bulge (Bensby et al. 2013), shown in orange. The best cluster candidates, selected on grounds of their kinematics and position, are highlighted in red, while blue symbols denote the two stars with GC properties except for deviating abundances.

disk, bulge and disk stars (Kunder et al. 2012; Ness et al. 2013; Zoccali et al. 2017). The MDF, however, lacks the pronounced peak at solar values with its strong tail far above solar metallicities, as is seen in low-latitude fields that are uniquely bulgedominated.

\subsection{Alpha-elements: Si, Ca, and Ti}

In Fig. 7 we compare the measurements of our GC and foreground stars with samples from the literature. Since the errors on our data are considerably large, we chose to consider in the following the straight average of the three explosive $\alpha$-elements $\mathrm{Si}, \mathrm{Ca}$, and $\mathrm{Ti}$ as a representative chemical tracer, even though this simplistic approach cannot account for differences in their exact production channels (e.g., Timmes et al. 1995; Venn et al. 2004).

For comparison, we use the solar neighborhood sample of Bensby et al. (2014, light gray points) and microlensed bulge dwarfs of Bensby et al. (2013, orange). There is a clear dichotomy in the data in the sense of the bulge stars having $[\alpha / \mathrm{Fe}]$ ratios that are higher by $0.1-0.2$ dex at subsolar metallicities, thereby vastly overlapping with the thick disk (see also 


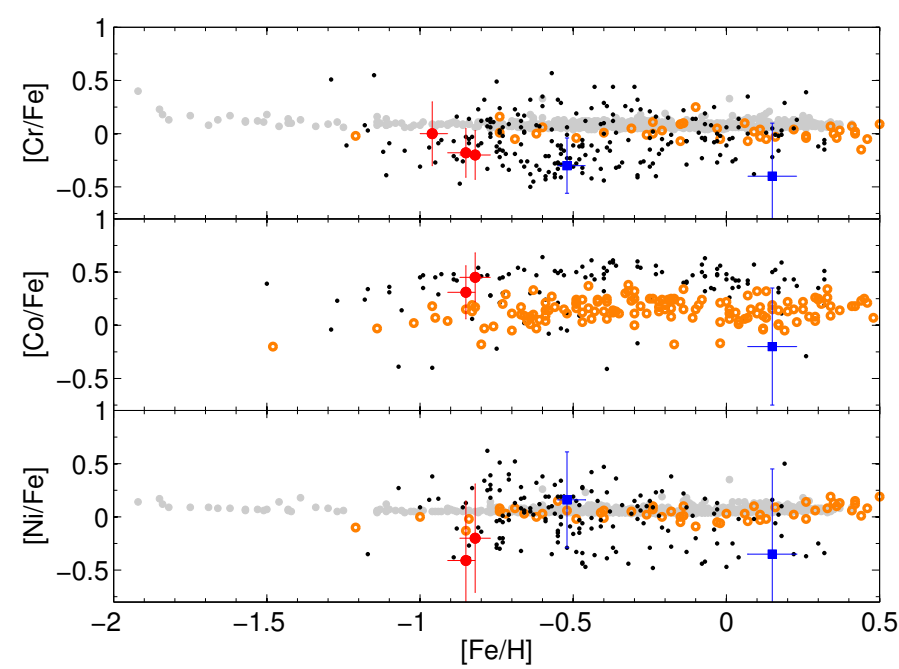

Fig. 8. Same as Fig. 7, but for the Fe-peak elements. Here, the Co-data are from Johnson et al. (2014).

Fulbright et al. 2007; McWilliam 2016). Taken at face value, the majority of our non-cluster stars at their higher $\alpha$-abundances are therefore more consistent with an association with the bulge population than with the disk. Fulbright et al. (2007) assigned these values to indications of supernovae II nucleosynthesis and pointed out the considerably lower scatter in the averaged $[\langle\mathrm{Si}$, $\mathrm{Ca}, \mathrm{Ti}\rangle / \mathrm{Fe}]$ ratio compared to the Galactic halo, for example, which suggests efficient mixing in the bulge.

About a third of the sample shows lower $[\alpha / \mathrm{Fe}]$ ratios that overlap with the solar neighborhood stars, down to values below zero. This renders the stars toward the sightline of ESO452 a representative mix of the expected components (see also Koch et al. 2016).

Two of the most "metal-poor" stars among the five GC candidates have slightly depleted $[\alpha / \mathrm{Fe}]$ ratios, while the third star is enhanced to halo-like values, resulting in an average for the three stars of 0.27 dex with a $1 \sigma$ scatter of 0.10 dex. This is compatible with the disk population and does not show the elevated levels of the bulge, although the large error bars make this distinction marginal. One star at $[\mathrm{Fe} / \mathrm{H}]=-0.5$ dex shares this abundance, but its higher metallicity renders it a likely foreground object. Finally, the solar-scaled $[\alpha / \mathrm{Fe}]$ ratio of most metal-rich star (number 56) confirms that it is a typical metal-rich disk star without any association with ESO452.

\subsection{Fe-peak elements: $\mathrm{Cr}$, Co, and $\mathrm{Ni}$}

The measurement of these abundances from just a few lines at most is very uncertain, and the resulting distribution (Fig. 8) of our stars show mainly a large scatter around the solar values, for both the foreground stars and the GC candidates. In contrast, the literature data from the high-resolution studies in the disk and bulge show a very low scatter and broadly overlap with each other. Owing to the large uncertainties and scatter of our measurements, we refrain from any strong conclusions on the enrichment of ESO452 in comparison to the Galactic components.

\subsection{Neutron-capture elements: Sr and Eu}

The blue part of the AAOmega spectra is plagued by low $\mathrm{S} / \mathrm{N}$ and most features are heavily blended. Thus we caution that the
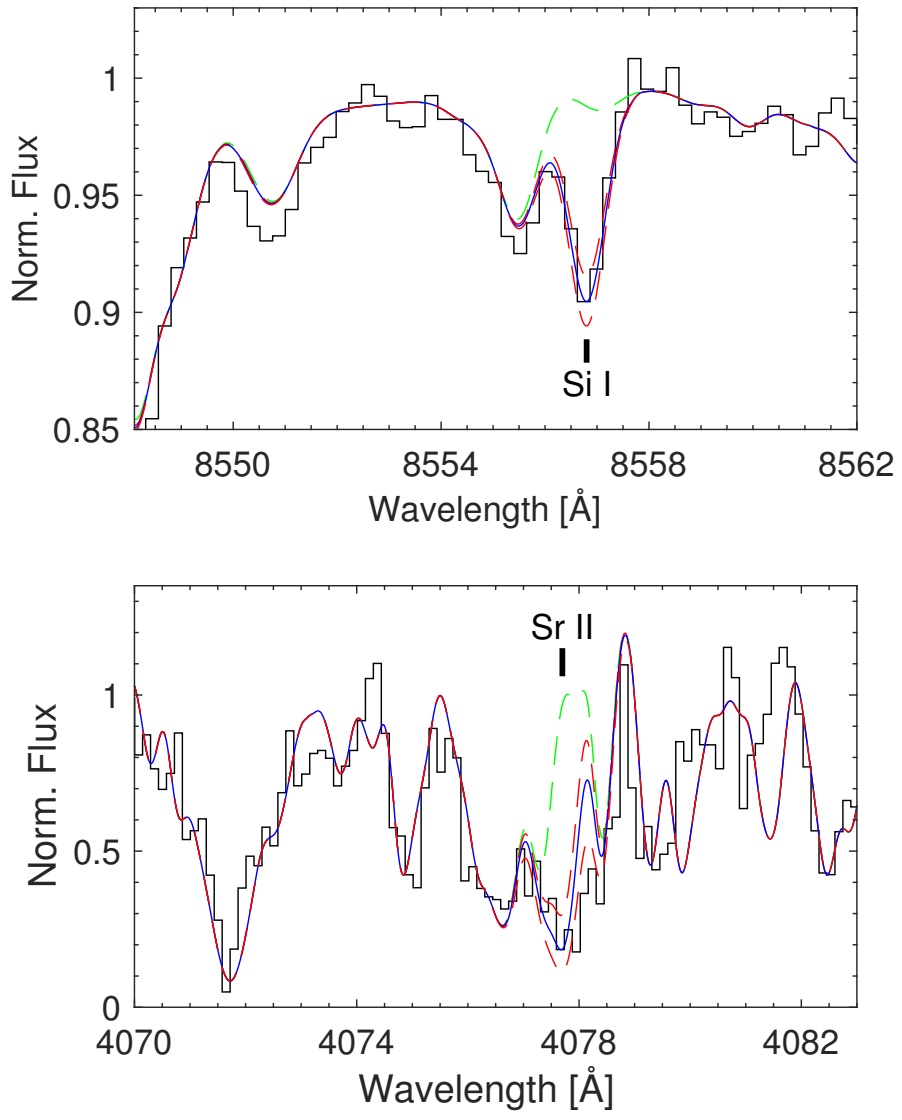

Fig. 9. Synthetic spectra around the Si $8556 \AA$-line (top) and the Sr $4077 \AA$ A-line (bottom panel) in star 204 (black histograms). The blue lines have been computed with the best-fit abundances of $[\mathrm{Si} / \mathrm{Fe}]=0.44$ and $[\mathrm{Sr} / \mathrm{Fe}]=0.00 \mathrm{dex}$, accounting for errors of $\pm 0.2 \mathrm{dex}$ (red). The green lines show syntheses without any $\mathrm{Si}$ and $\mathrm{Sr}$, respectively.

derived abundances are uncertain, and we impose a fitting uncertainty of \pm 0.15 dex that is due to continuum placement and blends alone. Moreover, since the heavy blending at the moderate resolution rendered measurements of abundances of the metal-rich foreground component impossible, we restricted our analysis of the blue spectra to the five GC member candidates.

The $\mathrm{Sr}$ abundance was derived from the blue spectra by virtue of the strong 4077 and $4215 \AA$ lines using spectral synthesis; since the spectral range also covered the wavelength of the two strongest blue Eu lines, we also attempted to derive abundances or place limits from the features at 4129 and $4205 \AA$.

To this end, we used Moog (Sneden 1973, version 2014) and Kurucz model atmospheres adopting the SP_ACE stellar parameters derived above. The line lists were optimized for the blue wavelength range using the Sneden et al. (2014) list that incorporates the newest hyperfine structure data for $\mathrm{Sr}$ (Bergemann et al. 2012; Hansen et al. 2013). In order to treat the continuum best, the blue spectra were normalized in two different ways using a cubic spline and Legendre polynomial. For star 204, which has the best S/N, this was straightforward. However, for stars 396 and 55, we adopted the average abundance from each of the two normalizations, which yielded results that agreed to within 0.1 dex. To further optimize the continuum placement, the synthesis was carried out using the full spectral range in pieces $100 \AA$ wide each (4050-4150 $\AA$ and 4150-4250 $\AA$ around the two $\mathrm{Sr}$ and Eu lines). This allowed us to derive $\mathrm{Sr}$ abundances in three stars, Eu in star 204, and one lower limit for Eu in star 


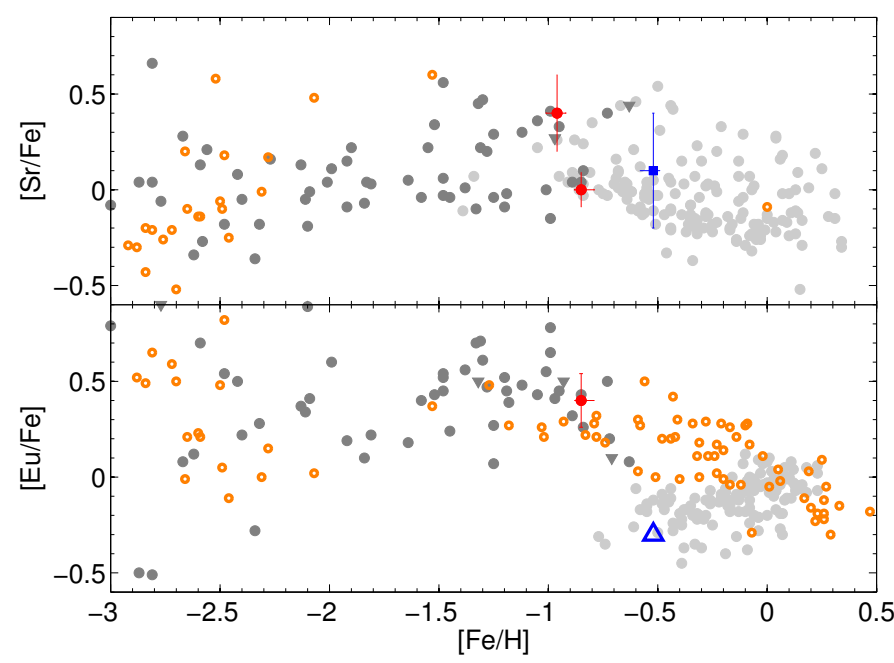

Fig. 10. Abundance ratios for the $n$-capture elements $\mathrm{Sr}$ and $\mathrm{Eu}$ with literature data. As before, gray points denote disk and halo stars of Battistini \& Bensby (2012, dark gray) and Battistini \& Bensby 2016, light gray) for Sr, and Hansen et al. (2012, dark gray) and Koch \& Edvardsson (2002, light gray) for Eu. Orange dots show the bulge component (Johnson et al. 2012), extending to the metal-poor bulge below $[\mathrm{Fe} / \mathrm{H}]<-2$ dex (Casey \& Schlaufman 2015; Howes et al. 2016; Koch et al. 2016).

396 due to the low S/N and line saturation. A section of of our observations and the best fit is shown in Fig. 9.

As can bee seen in Fig. 10, the derived Sr abundances are above solar and are found to be in agreement with the disk stars from Battistini \& Bensby (2016) and the mixed halo and disk sample of Hansen et al. (2012). Data for Sr in the Galactic bulge are only available for a few metal-poor field stars below -2 dex (Casey \& Schlaufman 2015; Howes et al. 2016; Koch et al. 2016), and our measurements provide the first $\mathrm{Sr}$ abundances in a metal-rich system toward the bulge.

The Eu abundance for one GC star is in turn compatible with the bulge population (Johnson et al. 2012), while the less reliable candidate, at $[\mathrm{Fe} / \mathrm{H}]=-0.5 \mathrm{dex}$, has a lower limit in agreement with either bulge or disk (Koch \& Edvardsson 2002). In light of the measurement uncertainties, we refrain from unambiguously assigning these targets to either Galactic component.

\section{Correlation with kinematics}

For an understanding of the origin and dynamical evolution of ESO452, it is of considerable interest to determine the orbit of this GC. However, at the distance of ESO452, it is nontrivial to obtain proper motions of sufficient quality for a direct calculation of the orbit. Nevertheless, we cross-matched the stars observed here with the HSOY ("Hot Stuff for One Year") proper motion catalog (Altmann et al. 2017) as well as the UCAC5 proper motion catalog (Zacharias et al. 2017). Both these catalogs capitalize on the exquisite positions provided in Gaia-DR1 (Gaia Collaboration 2016), providing proper motions much improved over the previously available catalogs.

We were able to match 341 of our observed stars $(94 \%$ of our sample) with those provided in HSOY and UCAC5, where two of these are probable GC members. Figure 11 shows that the proper motions of the two GC member candidates that are present in the catalog are slightly offset from the majority of the bulge field stars, in agreement with the finding that our GC candidates are distinct from the field and instead belong to
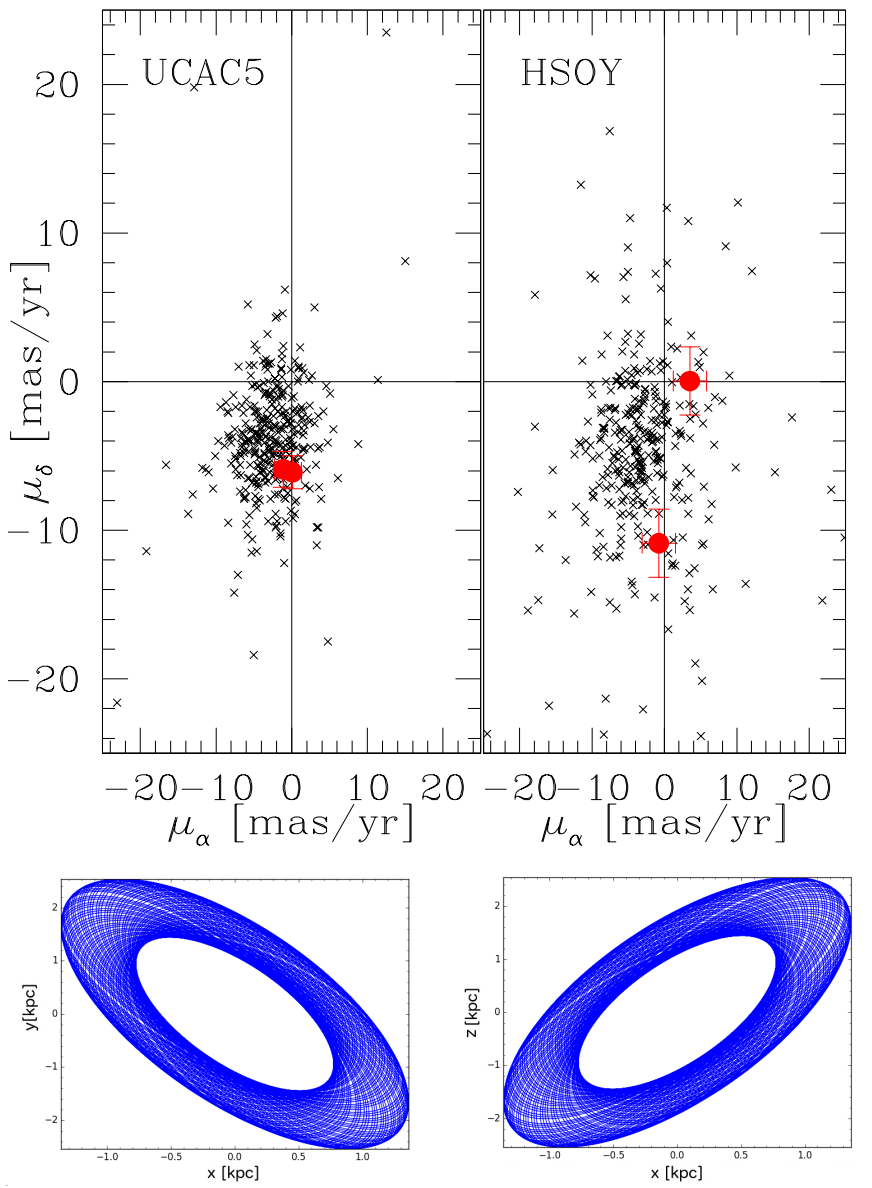

Fig. 11. Top: proper motions of our observed stars, where the solid red circles show the two GC candidates that are listed in both the HSOY and UCAC5 catalogs. Bottom: projections of the orbit of the two candidate ESO452 stars in the $x y$ and $x z$ plane. The orbits indicate that they are confined to the bulge.

a different system. The two candidate cluster stars have HSOY proper motions that differ by $2-3 \sigma$ from each other, indicating that if these two stars are indeed cluster members, the uncertainties in their proper motion should be larger than indicated in HSOY. In contrast, the UCAC5 proper motions, which have smaller formal uncertainties, indicate that our cluster candidates have almost identical proper motions with an average of $\left(\mu_{\alpha}, \mu_{\delta}\right)=(-0.6 \pm 1.1,-6.0 \pm 1.2)$ mas. We therefore conclude that the proper motions also indicate that our cluster candidates are members of ESO452.

Adopting our radial velocities, the Gaia positions, the UCAC5 proper motions, and a distance of $7.2 \mathrm{kpc}$ (Cornish et al. 2006), we calculated orbits using the python package galpy (Bovy 2015). The Galactic potential assumed was a threecomponent Milky Way-like potential for the orbital integration. The two candidate cluster members have orbits with ellipticities of $e \sim 0.3$, a maximum vertical height, $Z_{\max }$, of $\sim 2.5 \mathrm{kpc}$, and orbital pericenter $\left(r_{\min }\right)$ and apocenter $\left(r_{\max }\right)$ radii of $\sim 1.5 \mathrm{kpc}$ and $\sim 3 \mathrm{kpc}$, respectively, placing them well within the bulge region. Adopting a $1 \mathrm{kpc}$ larger cluster distance does not change the results significantly. In addition, using the slightly different proper motions of HSOY also returns orbits in which the stars are confined to the bulge. The orbital integration therefore indicates that this GC belongs to the bulge, in line with its abundance and velocity. 

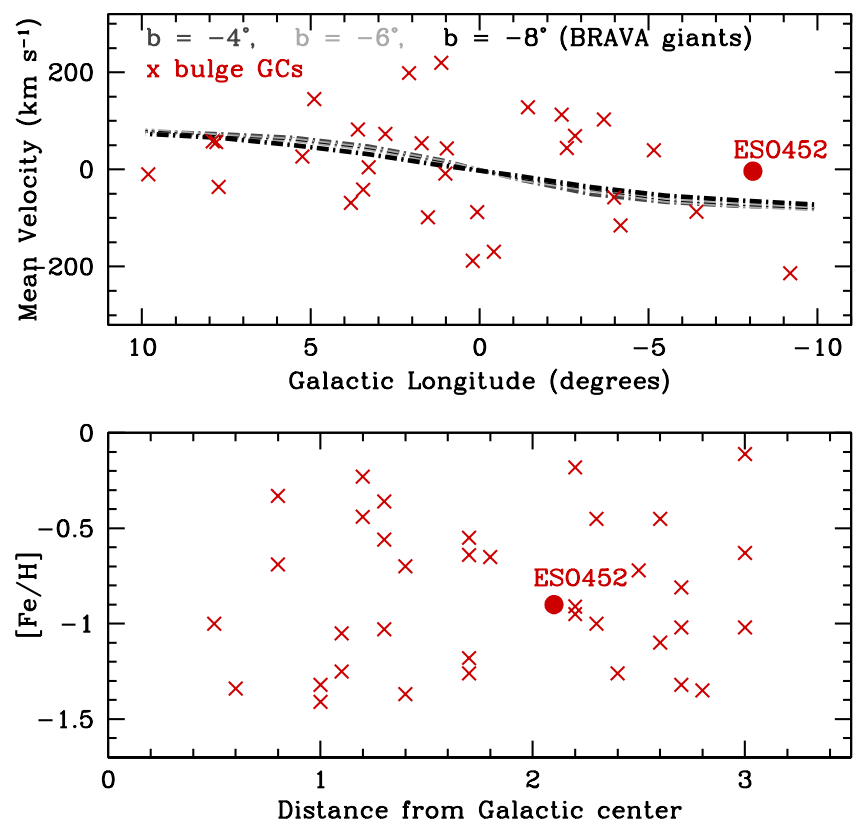

Fig. 12. Top: rotation curve for bulge stars (Kunder et al. 2012) and that of bulge GCs (Bica et al. 2016). Bottom: metallicities of bulge globular clusters. Overall, ESO452 has properties that are expected for a typical system in the Galactic bulge.

Turning to the Galactocentric velocity, this value corresponds to $-4.3 \pm 1.1 \mathrm{~km} \mathrm{~s}^{-1}$ (as determined by Eq. (1) in Howard et al. 2008). While this is a higher velocity than the mean stellar motion of the outer bulge (Kunder et al. 2012), it falls well within the range of velocities of GCs that belong to the bulge (Bica et al. 2016, and Fig. 12, top). In terms of its metallicity, it also shows a mean value that agrees with the values found among the bulge GCs, which is expected because they cover a range of more than $1 \mathrm{dex}$ in $[\mathrm{Fe} / \mathrm{H}]$. The mean Galactocentric velocity of our entire sample is $-60.7 \pm 4.7 \mathrm{~km} \mathrm{~s}^{-1}$ with a $1 \sigma$ dispersion of $79.7 \pm 3.3 \mathrm{~km} \mathrm{~s}^{-1}$. These values are well in line with what is expected of bulge field stars when we extrapolate the southern bulge rotation curve (e.g., Kunder et al. 2012; Ness et al. 2013) to this northern position on the sky (Fig. 12).

\section{No extra-tidal stars}

Our chemodynamical sample offers a unique opportunity of testing for signs of extra-tidal stars, which could have been stripped from the GC through tidal interactions while orbiting within the Galactic potential (e.g, Kunder et al. 2014a; Kuzma et al. 2016). To this end, we imposed a $3 \sigma$ cut around the cluster mean radial velocity to select stars outside the nominal tidal radius that share similar properties as the three bona fide member candidates characterized above (stars 55, 204, and 362).

As a result, we identify 15 stars within this kinematic cut in addition to the candidates within the tidal radius discussed above. Their location within our observations' field of view is shown in Fig. 13. As it stands, this would imply that these objects are preferentially aligned symmetrically around the GC, NW through SE. If these stars were indeed to represent a tidal extension of the GC, they should roughly follow the GC orbit around the Galactic center (e.g., Dehnen et al. 2004; Jordi \& Grebel 2010). However, as indicated in Fig. 13, no obvious correlation with direction of the Milky Way center can be established. Furthermore, these 15 stars show no coherence in proper motions or

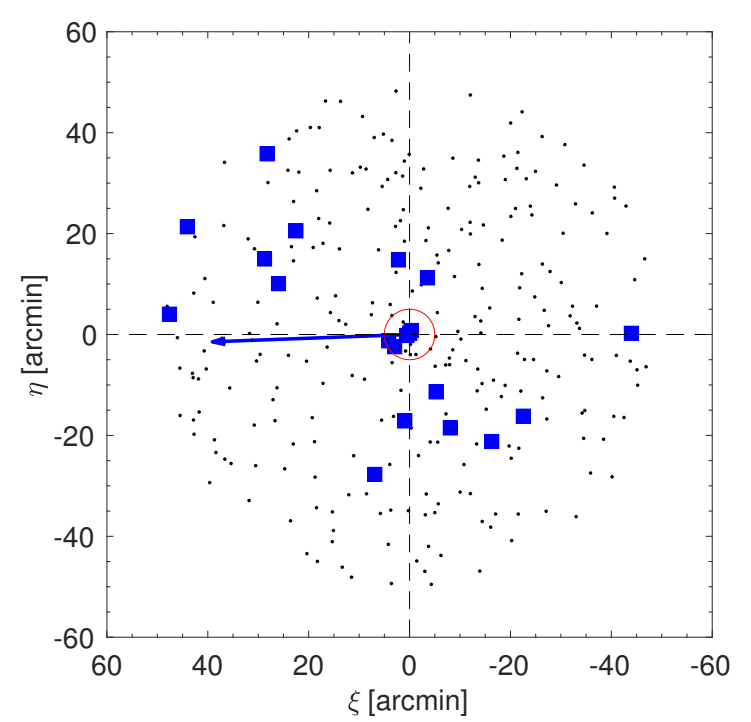

Fig. 13. Spatial location of all sample stars (black) and targets within $3 \sigma$ of the mean velocity of the GC candidates (blue squares). The blue arrow indicates the direction toward the Galactic center, and the red circle encompasses the nominal tidal radius.

abundance space and are instead found across the entire range in proper motion, metallicity, and $[\alpha / \mathrm{Fe}]$-ratios with no overlap with the three best GC candidates. We conclude that our measured velocities and abundances offer no evidence of tidally stripped material from ESO452.

\section{Discussion}

In contrast to remote and uniquely metal-poor GCs in the Galactic halo, stellar systems in the interface of the Milky Way disk, bulge, and halo are notoriously difficult to classify. By focusing on the chemical composition and dynamics of one of these elusive systems, the low-mass GC ESO452-SC11, we were able to gather significant information to place it in the context of the central regions of our Galaxy. Considering its moderately low metallicity of -0.88 dex and a Galactocentric velocity of $-4 \mathrm{~km} \mathrm{~s}^{-1}$, and folding in the chemical abundance ratios we obtained, we could establish strong evidence that this object is indeed associated with the Galactic bulge and is not a somewhat younger progeny of the inner halo. By identifying at least three bona fide GC member candidates, we showed that their $[\alpha / \mathrm{Fe}]$ ratios are consistent with a disk membership as well, while they are chemically marginally consistent with the slightly $\alpha$-enhanced bulge component. To be of avail, future high-resolution studies of proper motion pre-selected targets are indispensable.

ESO452 is a very good example of a low-mass metalintermediate $([\mathrm{Fe} / \mathrm{H}] \sim-1 \mathrm{dex}) \mathrm{GC}$ that resembles for instance the inner halo clusters NGC 6362 and NGC 6121 (Marino et al. 2008; Massari et al. 2017) in many regards. These are among the lowest-mass systems that show the presence of multiple stellar populations through light element variations, which remain undetected in ESO452 in our work, however. Still, the $\alpha$-element, $\mathrm{Cr}$, and Eu abundances in the bulge GC studied here agree well with those found in the above objects, and their other elemental abundances are also consistent within the uncertainties.

By considering the proper motions of the candidate members, we were able to consolidate a clear connection with the Galactic bulge in that this GC exhibits an elliptical orbit that confines it to the innermost $\sim 3 \mathrm{kpc}$ of the Galaxy. Despite the 
higher stellar density of the inner Galactic region, no evidence of any tidal happenings in the form of stars beyond the tidal radius were found in our wide-field data, which either indicates that any spatial signatures could have been erased during violent GC disruption, or that our data simply have not picked up any such "extra-tidal" component. The former is bolstered by the lack of kinematically cold streams in the bulge (Kunder et al. 2014b), however, which would be witnesses to ancient accretion events (cf. Hansen et al. 2016), as has also been proposed for ESO452 (Cornish et al. 2006) and as has indeed been found around several other systems (Kunder et al. 2014a).

Acknowledgements. Based on data acquired through the Australian Astronomical Observatory, via program NOAO Prop. ID: 2013A-0120 (PI: A. Kunder). C.J. Hansen acknowledges financial support by the Augustinus Foundation and the VILLUM Foundation (grant number VKR023371). We are grateful to the anonymous referee for a swift and constructive report.

\section{References}

Alonso, A., Arribas, S., \& Martinez-Roger, C. 1996, A\&A, 313, 873 Alonso, A., Arribas, S., \& Martínez-Roger, C. 1999, A\&AS, 140, 261 Altmann, M., Roeser, S., Demleitner, M., Bastian, U., \& Schilbach, E. 2017, A\&A, 600, L4

Armandroff, T. E., \& Zinn, R. 1988, AJ, 96, 92

Barklem, P. S., Christlieb, N., Beers, T. C., et al. 2005, A\&A, 439, 129

Battistini, C., \& Bensby, T. 2016, A\&A, 586, A49

Bensby, T., Yee, J. C., Feltzing, S., et al. 2013, A\&A, 549, A147

Bensby, T., Feltzing, S., \& Oey, M. S. 2014, A\&A, 562, A71

Bergemann, M., Hansen, C. J., Bautista, M., \& Ruchti, G. 2012, A\&A, 546, A90

Bica, E., Ortolani, S., \& Barbuy, B. 2016, PASA, 33, e028

Boeche, C., \& Grebel, E. K. 2016, A\&A, 587, A2

Bovy, J. 2015, ApJS, 216, 29

Carretta, E., Bragaglia, A., Gratton, R., D’Orazi, V., \& Lucatello, S. 2009, A\&A, 508,695

Casey, A. R., \& Schlaufman, K. C. 2015, ApJ, 809, 110

Chiba, M., \& Beers, T. C. 2000, AJ, 119, 2843

Cornish, A. S. M., Phelps, R. L., Briley, M. M., \& Friel, E. D. 2006, AJ, 131, 2543

Cutri, R. M., Skrutskie, M. F., van Dyk, S., et al. 2003, 2MASS All Sky Catalog of point sources

Dehnen, W., Odenkirchen, M., Grebel, E. K., \& Rix, H.-W. 2004, AJ, 127, 2753

Dotter, A., Chaboyer, B., Jevremović, D., et al. 2008, ApJS, 178, 89

Dubath, P., Meylan, G., \& Mayor, M. 1997, A\&A, 324, 505

Ferraro, F. R., Dalessandro, E., Mucciarelli, A., et al. 2009, Nature, 462, 483

Fulbright, J. P., McWilliam, A., \& Rich, R. M. 2007, ApJ, 661, 1152

Gaia Collaboration (Prusti, T., et al.) 2016, A\&A, 595, A1

Hansen, C. J., Primas, F., Hartman, H., et al. 2012, A\&A, 545, A31

Hansen, C. J., Bergemann, M., Cescutti, G., et al. 2013, A\&A, 551, A57

Hansen, C., Ludwig, H.-G., Seifert, W., et al. 2015, Astron. Nachr., 336, 665
Hansen, C. J., Rich, R. M., Koch, A., et al. 2016, A\&A, 590, A39 Harris, W. E. 1996, AJ, 112, 1487

Hendricks, B., Koch, A., Lanfranchi, G. A., et al. 2014, ApJ, 785, 102 Howard, C. D., Rich, R. M., Reitzel, D. B., et al. 2008, ApJ, 688, 1060 Howes, L. M., Asplund, M., Keller, S. C., et al. 2016, MNRAS, 460, 884 Jofré, P., Heiter, U., Soubiran, C., et al. 2014, A\&A, 564, A133 Johnson, C. I., Rich, R. M., Kobayashi, C., \& Fulbright, J. P. 2012, ApJ, 749, 175

Johnson, C. I., Rich, R. M., Kobayashi, C., Kunder, A., \& Koch, A. 2014, AJ, 148,67

Jordi, K., \& Grebel, E. K. 2010, A\&A, 522, A71

Kleyna, J. T., Wilkinson, M. I., Evans, N. W., \& Gilmore, G. 2004, MNRAS, 354, L66

Koch, A., \& Edvardsson, B. 2002, A\&A, 381, 500

Koch, A., \& McWilliam, A. 2008, AJ, 135, 1551

Koch, A., Côté, P., \& McWilliam, A. 2009, A\&A, 506, 729

Koch, A., Lépine, S., \& Çalışkan, Ş. 2012, in EPJ Web Conf., 19, 03002

Koch, A., McWilliam, A., Preston, G. W., \& Thompson, I. B. 2016, A\&A, 587, A124

Kunder, A., Koch, A., Rich, R. M., et al. 2012, AJ, 143, 57

Kunder, A., Bono, G., Piffl, T., et al. 2014a, A\&A, 572, A30

Kunder, A., Rich, R. M., Gerhard, O., et al. 2014b, in Formation and Evolution of the Galactic Bulge, proceedings of a conference held 20-24 January, at the Sexten Center for Astrophysics, 15

Kunder, A., Kordopatis, G., Steinmetz, M., et al. 2017, AJ, 153, 75

Kuzma, P. B., Da Costa, G. S., Mackey, A. D., \& Roderick, T. A. 2016, MNRAS, 461, 3639

Lauberts, A., Holmberg, E. B., Schuster, H.-E., \& West, R. M. 1981, A\&AS, 43, 307

Marín-Franch, A., Aparicio, A., Piotto, G., et al. 2009, ApJ, 694, 1498

Marino, A. F., Villanova, S., Piotto, G., et al. 2008, A\&A, 490, 625

Massari, D., Mucciarelli, A., Dalessandro, E., et al. 2017, MNRAS, 468, 1249

Mauro, F., Moni Bidin, C., Geisler, D., et al. 2014, A\&A, 563, A76

McWilliam, A. 2016, PASA, 33, e040

Ness, M., Freeman, K., Athanassoula, E., et al. 2013, MNRAS, 430, 836

Nissen, P. E., \& Schuster, W. J. 2010, A\&A, 511, L10

Pryor, C., \& Meylan, G. 1993, in Structure and Dynamics of Globular Clusters, eds. S. G. Djorgovski, \& G. Meylan, ASP Conf. Ser., 50, 357

Ruchti, G. R., Fulbright, J. P., Wyse, R. F. G., et al. 2010, ApJ, 721, L92

Schlafly, E. F., \& Finkbeiner, D. P. 2011, ApJ, 737, 103

Schlegel, D. J., Finkbeiner, D. P., \& Davis, M. 1998, ApJ, 500, 525

Sharples, R., Walker, A., \& Cropper, M. 1990, MNRAS, 246, 54

Shetrone, M. D., Siegel, M. H., Cook, D. O., \& Bosler, T. 2009, AJ, 137, 62

Siebert, A., Williams, M. E. K., Siviero, A., et al. 2011, AJ, 141, 187

Sneden, C., Lucatello, S., Ram, R. S., Brooke, J. S. A., \& Bernath, P. 2014, ApJS, 214, 26

Sneden, C. A. 1973, Ph.D. Thesis, University of Texas, Austin

Timmes, F. X., Woosley, S. E., \& Weaver, T. A. 1995, ApJS, 98, 617

Vásquez, S., Zoccali, M., Hill, V., et al. 2015, A\&A, 580, A121

Venn, K. A., Irwin, M., Shetrone, M. D., et al. 2004, AJ, 128, 1177

Warren, S. R., \& Cole, A. A. 2009, MNRAS, 393, 272

Zacharias, N., Finch, C., \& Frouard, J. 2017, AJ, 153, 166

Zoccali, M., Vasquez, S., Gonzalez, O. A., et al. 2017, A\&A, 599, A12

Zwitter, T., Castelli, F., \& Munari, U. 2004, A\&A, 417, 1055 